ARTICLE

Received 8 Aug 2016 | Accepted 25 Nov 2016 | Published 4 Jan 2017

\title{
Evolution of form in metal-organic frameworks
}

Jiyoung Lee ${ }^{1}$, Ja Hun Kwak ${ }^{2} \&$ Wonyoung Choe $^{1}$

Self-assembly has proven to be a widely successful synthetic strategy for functional materials, especially for metal-organic materials (MOMs), an emerging class of porous materials consisting of metal-organic frameworks (MOFs) and metal-organic polyhedra (MOPs). However, there are areas in MOM synthesis in which such self-assembly has not been fully utilized, such as controlling the interior of MOM crystals. Here we demonstrate sequential self-assembly strategy for synthesizing various forms of MOM crystals, including doubleshell hollow MOMs, based on single-crystal to single-crystal transformation from MOP to MOF. Moreover, this synthetic strategy also yields other forms, such as solid, core-shell, double and triple matryoshka, and single-shell hollow MOMs, thereby exhibiting form evolution in MOMs. We anticipate that this synthetic approach might open up a new direction for the development of diverse forms in MOMs, with highly advanced areas such as sequential drug delivery/release and heterogeneous cascade catalysis targeted in the foreseeable future.

\footnotetext{
${ }^{1}$ Department of Chemistry, Ulsan National Institute of Science and Technology, Ulsan 44919, Republic of Korea. ${ }^{2}$ Department of Chemical Engineering, Ulsan National Institute of Science and Technology, Ulsan 44919, Republic of Korea. Correspondence and requests for materials should be addressed to J.H.K. (email: jhkwak@unist.ac.kr) or to W.C. (email: choe@unist.ac.kr).
} 
$\mathrm{F}$ or the past few decades, a main theme of materials chemistry has been to control the exterior and interior of solidstate materials to meet the rising demand for new, highperformance functional materials ${ }^{1-6}$. In particular, controlling the interior of the particles and thereby transforming solid particles into more sophisticated forms, such as core-shell, hollow, matryoshka (for example, Russian doll), yolk-shell and multi-shell hollow particles, has been an important synthetic challenge for materials chemists because these exotic materials could exhibit advanced functional properties in catalysis ${ }^{7-10}$, chemical sensing ${ }^{1-13}$, energy ${ }^{14-17}$ and biomedical applications ${ }^{18-21}$ (Fig. 1). Recently, similar synthetic efforts have been applied to metal-organic frameworks (MOFs), an emerging class of porous materials ${ }^{22-36}$. However, unlike other micro-/nanostructures reported in metal/metal oxide systems, those in MOFs are still in their infancy, as evidenced by the fact that the vast majority of these materials have hollow forms, an early stage of form evolution (Fig. 1) $)^{37}$. The synthetic strategies identified for hollow MOFs include templating methods (using polystyrene beads ${ }^{25,26}$, emulsion droplets ${ }^{27}, \mathrm{CO}_{2}$ bubbles $^{28}, \mathrm{MOFs}^{29}$ and metal-organic polyhedron (MOP) crystals $^{30}$ ), interfacial growing methods ${ }^{31}$, spray-drying techniques ${ }^{32}$ and surface-driven mechanisms ${ }^{33}$. A recent notable example from Lah group utilizes MOP crystals as a sacrificial template to synthesize hollow $\mathrm{MOFs}^{30}$. Despite these efforts, making further sophisticated forms in MOFs is significantly hampered by a lack of rational synthetic strategies.

Here, we first demonstrate a synthetic strategy for doubleshell hollow MOF via sequential self-assembly. The sequential steps involved in creating double-shell hollow MOF are shown in Fig. 2: (1) single-crystal to single-crystal transformation from MOP to MOF through postsynthetic linker insertion (I-a), (2) epitaxial growth of MOP on the MOF surface (I-b), (3) insertion of another linker to form double to triplematryoshka metal-organic materials (MOMs) (I-c), and finally (4) elimination of MOP by chemical etching (I-d). Through this stepwise synthetic procedure, we successfully complete form evolution from a parent MOP to various MOMs, including solid, core-shell, double and triple matryoshka, and hollow single- and double-shell structures.

\section{Results}

Synthesis of cuboctahedron MOP and its solid-state structure. Our journey to form evolution in MOMs begins with cuboctahedron MOPs (hereafter, cuo-MOPs). These MOPs are composed of $12 \mathrm{Cu}_{2}(\mathrm{COO})_{4}$ paddlewheel nodes and 24 5-R-1,3benzenedicarboxylic acid (R-mBDC, $\mathrm{R}=\mathrm{H}, \mathrm{OH}, \mathrm{NO}_{2}, \mathrm{SO}_{3}^{-}$ and so on $)^{38,39}$ (Fig. 3a), and some of these MOPs prefer face-centered cubic (fcc) packing. As reported by Eddaudi and $\mathrm{Lah}^{40,41}$, there is a striking structural similarity between such fcc-packed cuo-MOPs and their corresponding linkerinserted MOFs with ubt topology (Fig. 3b,c). The ubt-MOFs can be obtained by connecting the axial position of paddlewheel nodes in cuo-MOPs with linear linkers, such as 1,4-diazabicyclo[2.2.2] octane (dabco) and 4,4'-bipyridyl (bpy) through conventional solvothermal synthesis (Fig. 3c). For example, Chun et al. reported ubt-MOFs by connecting the twelve $\mathrm{Zn}$ (II) paddlewheel nodes of a MOP cage with their neighbouring MOPs via dabco, ${ }^{42}$ while Wang group synthesized $\mathrm{Cu}(\mathrm{II})$ paddlewheel-based ubt-MOFs using aminofunctionalized cuo-MOPs and bpy linkers ${ }^{43}$. Although there is no report demonstrating single-crystal to single-crystal transformation from cuo-MOP to ubt-MOF, we hypothesize that by careful choice of cuo-MOP, a structural transformation from fcc-packed cuo-MOP to ubt-MOF might be possible via postsynthetic insertion of dabco linkers ${ }^{44-46}$.

To connect adjacent cuo-MOPs with dabco linkers, the ideal $\mathrm{Cu}-\mathrm{Cu}$ distance between the two paddlewheel nodes is estimated as 6.6-7.5 $\AA$ based on a Cambridge Structure Database search (Supplementary Table 1). A new member of the fcc-packed cuo-MOP family, UMOM-1 [ $\mathrm{Cu}_{24}(\mathrm{OH}-\mathrm{mBDC})_{24}$ $\left.(\mathrm{DMSO})_{8}\left(\mathrm{H}_{2} \mathrm{O}\right)_{16}\right]$ has been synthesized and characterized by $\mathrm{X}$-ray single-crystal diffraction, exhibiting a $\mathrm{Cu}-\mathrm{Cu}$ distance of $7.5-8.2 \AA$, which is slightly longer than the ideal distance for dabco insertion (Supplementary Figs 1-4 and Supplementary Data 1) due to a slight rotation of the MOP cages. Although the cages are not perfectly aligned for dabco insertion, the packing of the MOP cages are close to ideal fcc packing, which is a desired feature for the planned structural transformation (Fig. 3d).

Single-crystal to single-crystal transformation of MOP to MOF. When we soaked the crystals of UMOM-1 in a N,N'-dimethylformamide (DMF)/dimethyl sulfoxide (DMSO) solution $(\mathrm{v} / \mathrm{v}=1: 1)$ containing dabco, after $12 \mathrm{~h}$, we noticed a crystal colour change from blue to green without any visual cracks in the optical microscopic images (Fig. $3 \mathrm{e}$ and Supplementary Fig. 5). Subsequent X-ray single-crystal analysis confirmed that this is indeed a single-crystal to single-crystal transformation from MOP (UMOM-1) to MOF (UMOM-2) (Supplementary Fig. 6, Supplementary Table 2 and Supplementary Data 2). When we compared the structures of UMOM-1 and UMOM-2, the space group was changed from $I 4 / m$ to $F m-3 m$ with $0.87 \%$ cell volume reduction (Supplementary Tables 3 and 4 ). In UMOM-2, as expected, the cofacial paddlewheel nodes are connected by dabco with a $\mathrm{Cu}-\mathrm{Cu}$ distance of $7.0 \AA$ (Supplementary Figs 7 and 8), which is a significant decrease from those of 7.5 and $8.2 \AA$ in the parent MOP, UMOM-1. ${ }^{1} \mathrm{H}$ NMR spectra was obtained from digested solution of UMOM-2 to confirm the insertion of dabco (Supplementary Fig. 9). The ratio of OH-mBDC to dabco is $4.00: 1.02$, which agrees well with the ratio found in the $\mathrm{X}$-ray single-crystal analysis.

When the structural transformation from UMOM-1 to UMOM-2 was monitored by X-ray synchrotron powder diffraction (Fig. 3f and Supplementary Fig. 10) at the Pohang Accelerator Laboratory (PAL) in the Republic of Korea, noticeable $2 \theta$ changes could be seen at $9.6^{\circ}, 9.8^{\circ}$ and $10.7^{\circ}$, which correspond to (2-13), (222) and (3-12) reflections, respectively, for the parent UMOM-1. During the transformation, the intensity of the (2-13) reflection decreased, while the intensity of the (222) reflection increased with a slight shift toward higher $2 \theta$ values. Owing to cell parameter changes, the (3-12) reflection

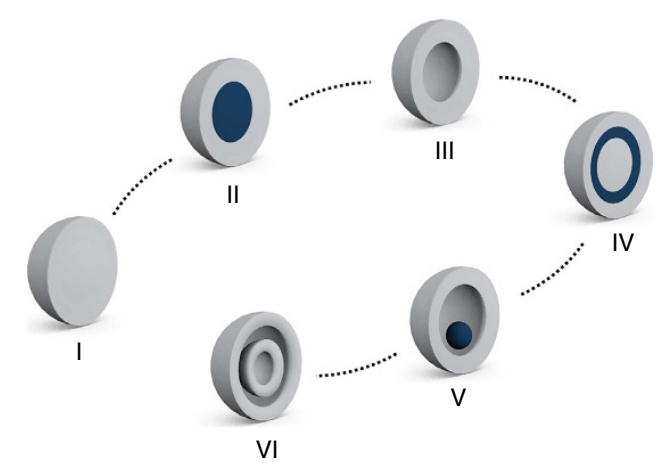

Figure 1 | Schematic representation of various forms of micro-/ nanostructures. Solid, core-shell, hollow, matryoshka, yolk-shell and multi-shell hollow structures (from I to VI, respectively). 


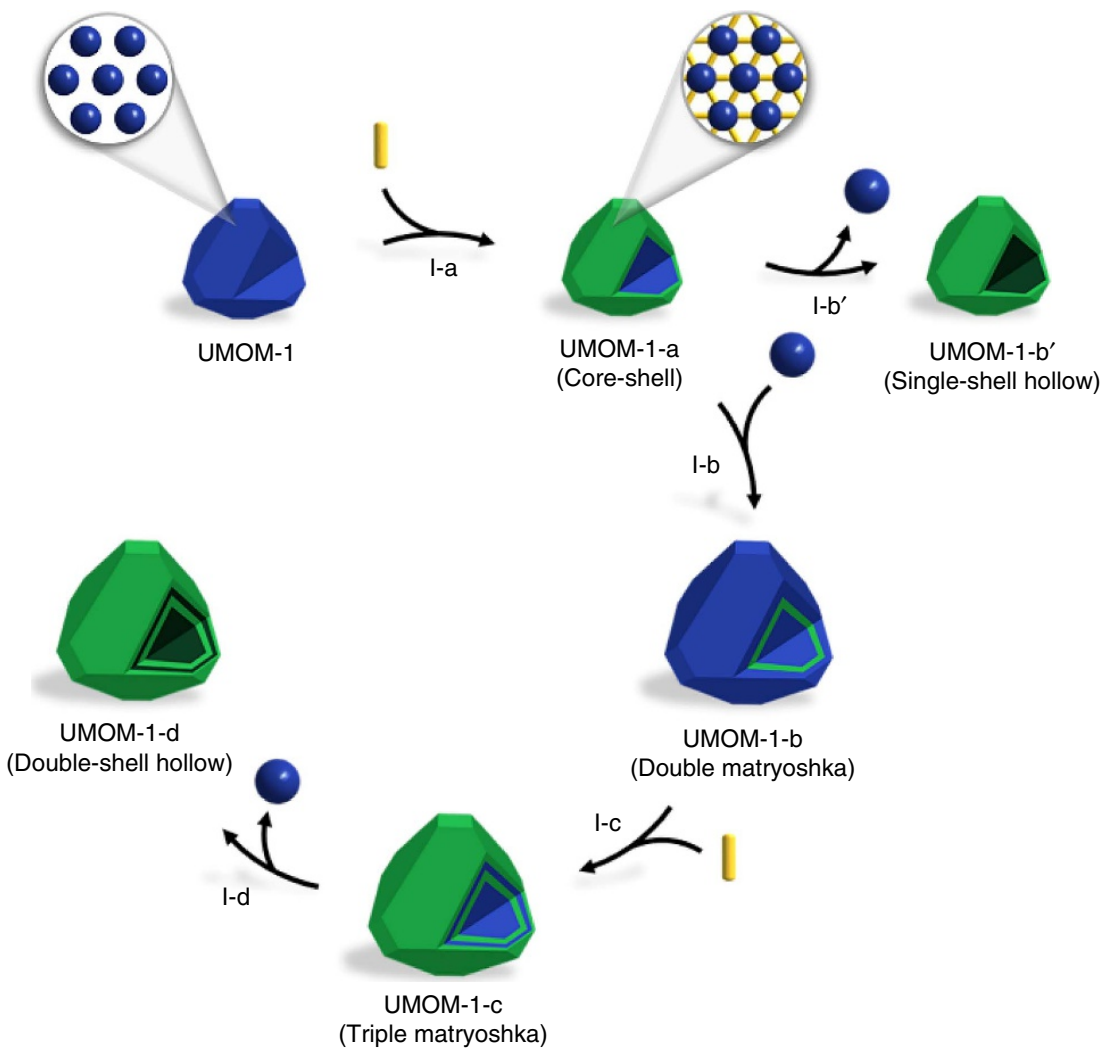

Figure 2 | Schematic illustration of form evolution. The process of synthesizing various forms of MOMs, including UMOM-1-a, UMOM-1-b, UMOM-1-b', UMOM-1-c and UMOM-1-d from UMOM-1. Stage I-a, UMOM-1 (MOP) $\rightarrow$ UMOM-1-a (core-shell) by partial postsynthetic linker insertion. Stage I-b', UMOM-1-a $\rightarrow$ UMOM-1-b' (single-shell hollow) by etching process. Stage I-b, UMOM-1-a $\rightarrow$ UMOM-1-b (double matryoshka) by epitaxial growth of UMOM-1 on the surface of UMOM-1-a. Stage I-c, UMOM-1-b $\rightarrow$ UMOM-1-c (triple matryoshka) by partial postsynthetic linker insertion. Stage I-d, UMOM-1-c $\rightarrow$ UMOM-1-d (double-shell hollow) by etching process. Blue sphere represents cuo-MOP and yellow rod represents dabco linker.

shifted to lower $2 \theta$ values. These results clearly indicate that there is a progressive change from UMOM-1 to UMOM-2 during the linker insertion reaction.

To propose a plausible mechanism for structural change, the transformation can be simulated using both the rotation and translation of MOP cages (Supplementary Fig. 11). When compared with the experimental X-ray synchrotron powder diffraction patterns, a $8.9^{\circ}$ clockwise rotation can occur along the c-axis, together with a $0.18 \AA$ translation inward (Supplementary Figs 12 and 13). From these results, we conclude that both rotation and translation of MOP cages are necessary to accommodate dabco molecules inside UMOM-1.

The resulting MOF, UMOM-2, is a porous material, as confirmed by the $\mathrm{N}_{2}$ sorption isotherm at $77 \mathrm{~K}$ (Supplementary Figs 14 and 15) with Brunauer-Emmett-Teller (BET) and Langmuir surface areas of 2,540 and $2,820 \mathrm{~m}^{2} \mathrm{~g}^{-1}$, respectively. The calculated surface area (Connolly surface) is $3,030 \mathrm{~m}^{2} \mathrm{~g}^{-1}$, as determined with a $1.4 \AA$ van der Waals scale factor and $1.84 \AA$ Connolly radius using Material Studio. The pore size distribution of UMOM-2 from the $\mathrm{N}_{2}$ isotherm using the oxide surface cylindrical model shows three different types of pores: $10.9,15.6$ and $18.8 \AA$, corresponding to the pore diameters of three types of cages (truncated tetrahedron, cuboctahedron and truncated octahedron, respectively, as identified in single-crystal structures) (Supplementary Fig. 16). $\mathrm{CO}_{2}$ adsorption-desorption isotherm was also obtained, and the maximum adsorbed amount of $\mathrm{CO}_{2}$ is $3.4 \mathrm{mmolg}^{-1}$ at $298 \mathrm{~K}$ and $6.8 \mathrm{mmol} \mathrm{g}^{-1}$ at $273 \mathrm{~K}$ (Supplementary Fig. 17). These values are comparable to those of rht-MOF-7 $\left(4.0 \mathrm{mmolg}^{-1}\right.$ at $298 \mathrm{~K}^{4}$ and $6.5 \mathrm{mmolg}^{-1}$ at $273 \mathrm{~K})^{47}$, also known as $\mathrm{Cu}$-TDPAT ${ }^{48}$, a leading MOF for
$\mathrm{CO}_{2}$ capture with rht topology, which has structural similarity with ubt topology ${ }^{41,49}$. The X-ray powder diffraction patterns show that the sample does not undergo any phase changes after activation (Supplementary Fig. 18). The peak positions of as-synthesized UMOM-2 and activated UMOM-2 match exactly. Thermogravimetric analysis shows that UMOM-2 is thermally stable up to $\sim 250^{\circ} \mathrm{C}$ (Supplementary Fig. 19).

The kinetic profile of the transformation procedure was obtained using ${ }^{1} \mathrm{H}$ NMR analysis (Supplementary Fig. 20). The kinetic profile shows that the process becomes slower over time.

Synthesis of single-crystal single-shell hollow MOF. While synthesizing UMOM-2, instead of making a solid MOF, we quenched the reaction during linker insertion, resulting in a core-shell system of MOF@MOP (from shell to core), which we call UMOM-1-a (Fig. 2). Such hybrid core-shell systems between MOP and MOF have not been established previously. Interestingly, we note that UMOM-2 is not soluble in methanol while UMOM-1 is. This is due to a drastic solubility change as a result of forming an extended solid. This drastic difference in solubility makes methanol an ideal solvent for chemical etching to create a hollow crystal. In other words, methanol can dissolve only MOP in the core-shell system of UMOM-1-a, leaving MOF in the outer shell untouched. When we treated UMOM-1-a with methanol, single-shell hollow crystals formed $\left(\mathrm{UMOM}-1-\mathrm{b}^{\prime}\right)$. Figure 4 and Supplementary Movies 1 and 2 clearly show the formation of single-shell hollow crystals of UMOM-1-b'. Interestingly, the thickness of the shell could be 
a

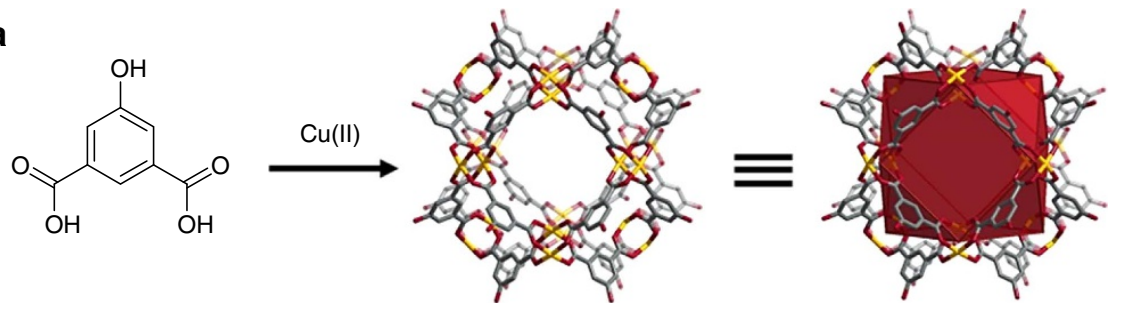

b

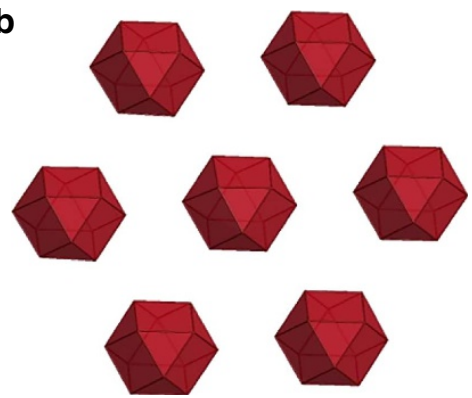

C

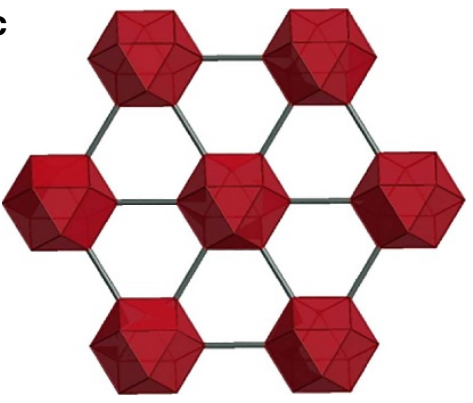

d
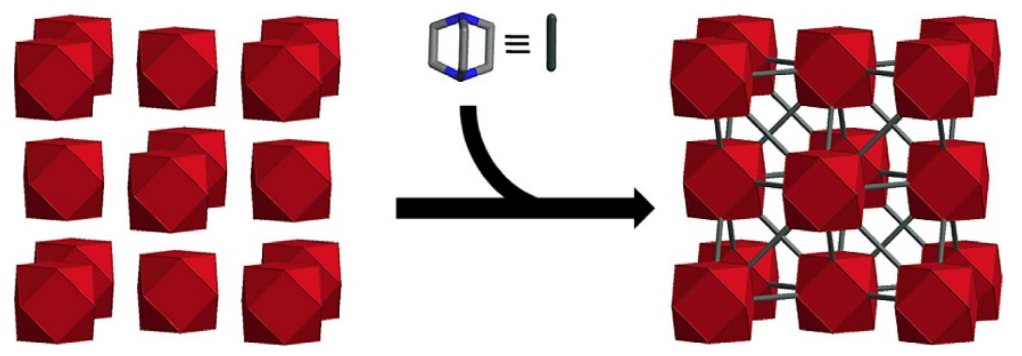

e

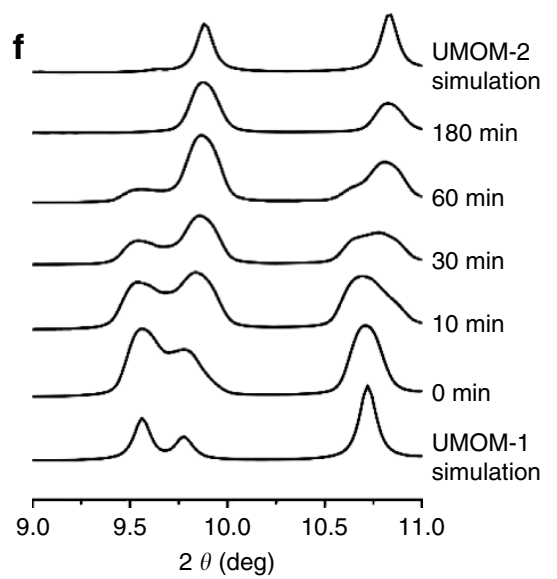

Figure 3 | Structural similarity between ubt-MOF and fcc-packed cuo-MOP. (a) The building blocks for construction of cuo-MOP; OH-mBDC as an organic linker and $\mathrm{Cu}_{2}(\mathrm{COO})_{4}$ paddlewheel as a metal node. $\mathrm{Cu}$, orange; $\mathrm{C}$, grey; $\mathrm{O}$, red; all hydrogen and solvents on the $\mathrm{Cu}(\mathrm{II})$ paddlewheel are omitted for clarity. Perspective view of the 3-fold axis of (b) fcc-packed cuo-MOP (UMOM-1) and (c) ubt-MOF (UMOM-2). Grey line represents linear dabco. (d) Scheme of single-crystal to single-crystal transformation from UMOM-1 to UMOM-2. (e) Photographs of the single crystal before (top of panel, UMOM-1) and after (bottom of panel, UMOM-2) structural transformation. (f) Monitoring the structural transformation using X-ray synchrotron powder diffraction. The crystals were collected by quenching during linker insertion reaction time at $0,10,30,60$ and 180 min.

controlled by the reaction time (t) for linker insertion (Fig. 5a-c). The average shell thickness is 10,26 and $40 \mu \mathrm{m}$ at $t=30,90$, and $120 \mathrm{~min}$, respectively. Optical microscopic images and scanning electron microscope (SEM) images clearly show the hollow interior of UMOM-1-b' (Fig. 4a-f and Supplementary Fig. 21). Ultraviolet-visible absorbance spectra show that the copper ions are released from the crystals to the methanol solution during UMOM-1-b' synthesis and etching reaction is completed in 200 min (Supplementary Figs 22 and 23). ${ }^{1} \mathrm{H}$ NMR analysis of the digested methanol solution shows that only $\mathrm{OH}-\mathrm{mBDC}$ of
MOP is released but dabco is not (Supplementary Fig. 24). All these results pinpoint that dissolved MOP is released from the crystals during the etching process.

Surprisingly, single-shell UMOM-1- $\mathrm{b}^{\prime}$ is indeed single crystalline, as determined by X-ray single-crystal diffraction (Supplementary Figs 25 and 26 and Supplementary Data 3). We were able to collect several sets of single-crystal data for singleshell MOFs, and these data were successfully refined (Supplementary Table 5). In addition, the porosity of UMOM$1-\mathrm{b}^{\prime}$ is maintained, as confirmed by $\mathrm{N}_{2}$ sorption, with the BET 
a

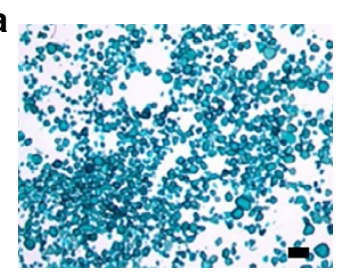

d

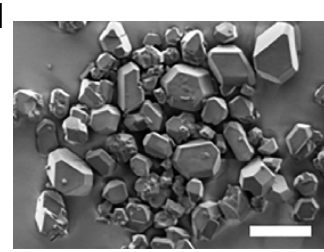

b

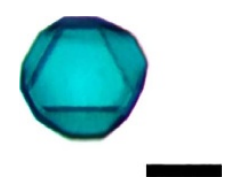

C
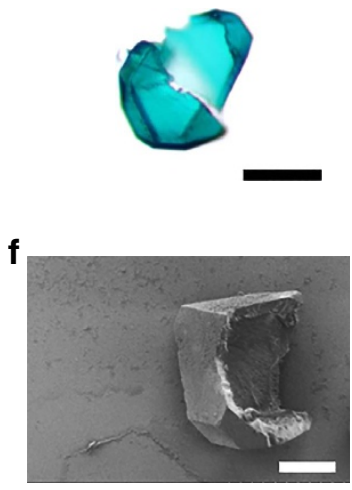

g
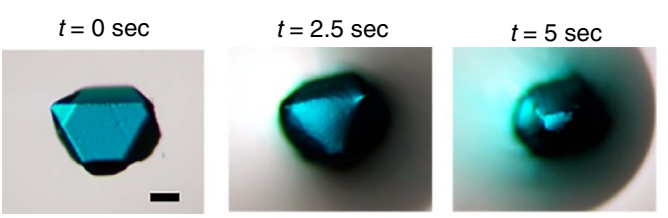

$t=7.5 \mathrm{sec}$
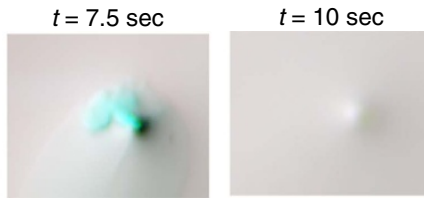

h

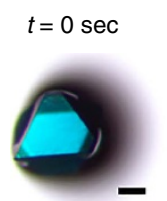

$t=30 \mathrm{sec}$

$t=60 \mathrm{sec}$

$t=90 \mathrm{sec}$

$t=120 \mathrm{sec}$
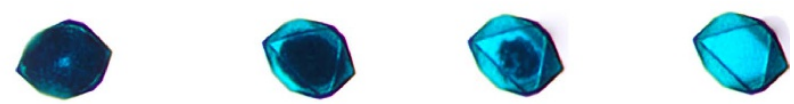

i

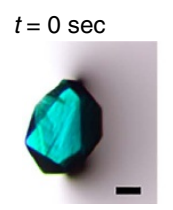

$t=75 \mathrm{sec}$

$t=150 \mathrm{sec}$

$t=225 \mathrm{sec}$

$t=300 \mathrm{sec}$
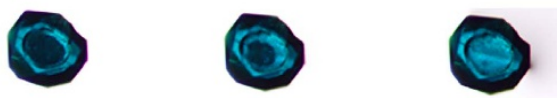

Figure 4 | Microscopic images of single-shell hollow UMOM-1-b'. (a) Optical microscopic image of overview of UMOM-1-b'. Scale bar, 500 $\mu$ m. $(\mathbf{b}, \mathbf{c})$ Optical microscopic images of single crystal of UMOM-1-b' before and after crack using needle tip. Scale bar, $200 \mu \mathrm{m}$. (d) SEM image of overview of UMOM-1-b'. Scale bar, $100 \mu \mathrm{m}$. (e) SEM image of single crystal of UMOM-1-b'. Scale bar, $50 \mu \mathrm{m}$. (f) SEM image of cracked single crystal of UMOM-1-b'. Scale bar, $50 \mu \mathrm{m}$. Time course images: (g) Single crystal of UMOM-1 in methanol. Single crystal of UMOM-1-b' in methanol with different linker insertion time (h) $10 \mathrm{~min}$, and (i) $90 \mathrm{~min}$. Scale bar, $200 \mu \mathrm{m}(\mathbf{g}-\mathbf{i})$. Linker insertion time is $30 \mathrm{~min}(\mathbf{a}-\mathbf{c})$, and $10 \mathrm{~min}(\mathbf{d}-\mathbf{f})$.

and Langmuir surface areas of 2,390 and $2,700 \mathrm{~m}^{2} \mathrm{~g}^{-1}$, respectively, which are slightly smaller than those for the solid MOF (Supplementary Figs 27 and 28). The pore size distribution of UMOM-1- $\mathrm{b}^{\prime}$ determined from the $\mathrm{N}_{2}$ isotherm using the oxide surface cylindrical model shows three different types of pores: 10.9, 15.6 and $18.8 \AA$, similar to those for the solid MOF counterpart (Supplementary Fig. 29).

Synthesis of double-shell hollow MOF. A double-shell hollow MOF was achieved by applying epitaxial growth of MOP to the crystal surface of core-shell UMOM-1-a. After soaking the crystals of UMOM-1-a in a DMSO/DMF mixture, a methanol solution containing $\mathrm{Cu}(\mathrm{II})$ and $\mathrm{OH}-\mathrm{mBDC}$ was added to make double-matryoshka UMOM-1-b (MOP@MOF@MOP). The same procedure was repeated one more time to evenly grow MOP on the seed MOF crystals. We obtained a shell growth image from SEM analysis of the crystals during the epitaxial growth reaction (Fig. 6a). The optical microscopic images show the well-coated single-crystalline shell of UMOM-1-b (Fig. 6b). Triple-matryoshka UMOM-1-c (MOF@MOP@MOF@MOP) was obtained by quenching during linker insertion into UMOM1-b. Finally, the double-shell UMOM-1-d was achieved using the same chemical etching method as used for single-shell UMOM-1-b' (Fig. 6c). Optical microscopic images confirm the empty space between the two shells of UMOM-1-d (Fig. 6d,e). Focused ion beam-SEM (FIB-SEM) images also confirm the empty space after milling the surface of UMOM-1-d using a $\mathrm{Ga}$ ion beam (Supplementary Method). The cross-section of UMOM-1-d represents the two crystalline shells and the cavity of the double-shell hollow MOF (Fig. 6f,g), demonstrating the double-shell nature of UMOM-1-d.

Unlike UMOM-1-a, -b and -c, UMOM-1-d shows permanent porosity, as confirmed by the $\mathrm{N}_{2}$ sorption with the BET and Langmuir surface areas of 2,150 and $2,540 \mathrm{~m}^{2} \mathrm{~g}^{-1}$, respectively, which are comparable to those for the single-shell hollow MOF (Supplementary Fig. 30). Thermogravimetric analysis shows that UMOM-1-a, -b, -c and -d are thermally stable up to $230-250^{\circ} \mathrm{C}$ (Supplementary Fig. 31 ).

\section{Discussion}

In summary, we successfully demonstrated the first example of double-shell hollow MOF, UMOM-1-d, via sequential self-assembly, followed by self-disassembly. In addition, this strategy leads to complete form evolution from a parent MOP to 

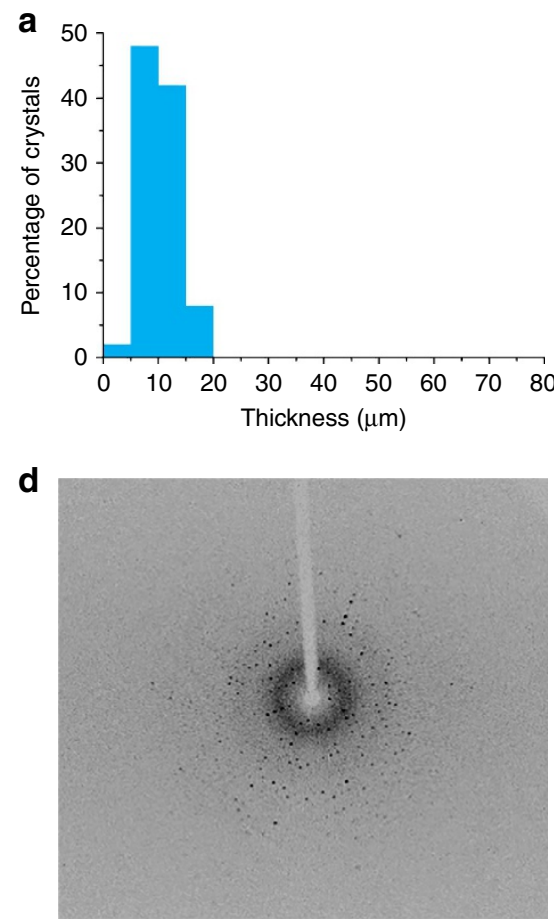
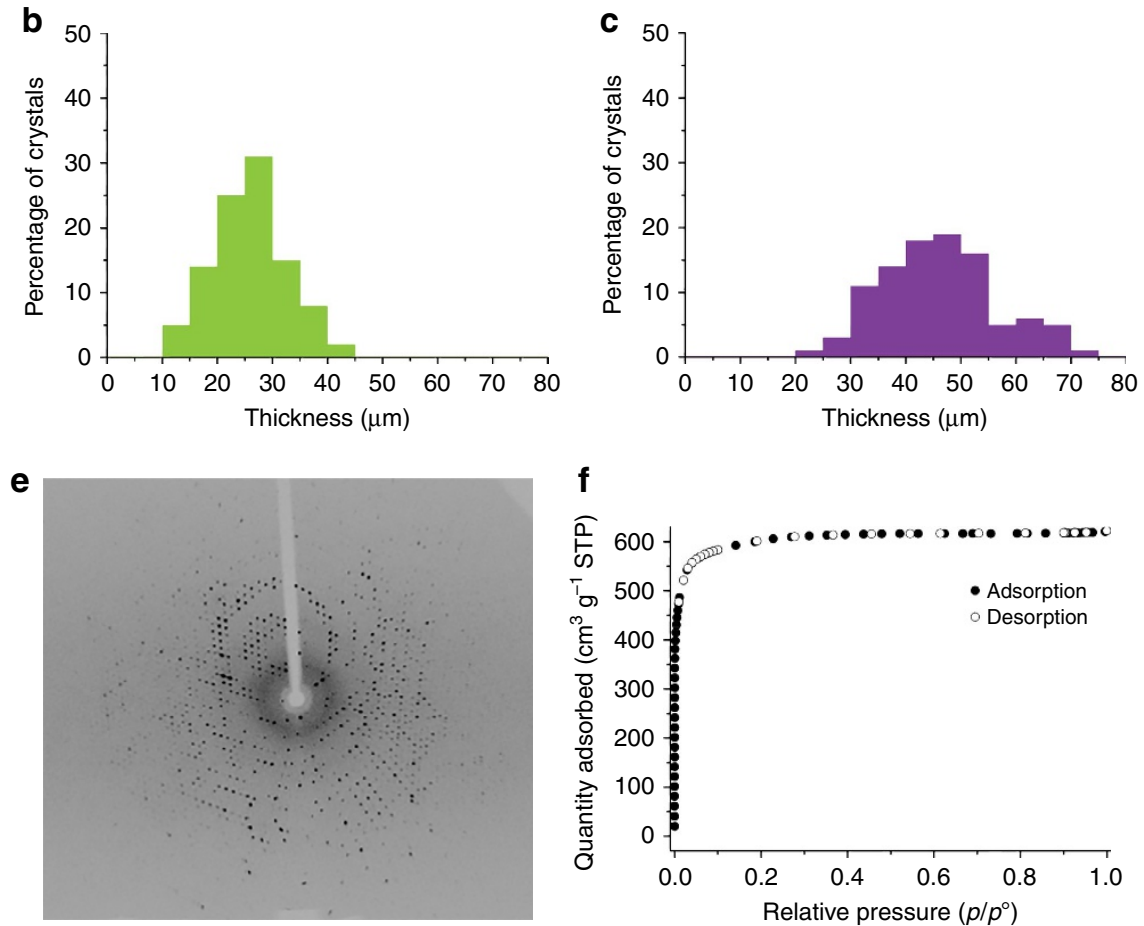

Figure 5 | Characteristics of single-shell hollow UMOM-1-b'. Shell thickness distribution of UMOM-1-b' depending on the different linker insertion time: (a) $30 \mathrm{~min}$, (b) $90 \mathrm{~min}$ and (c) $120 \mathrm{~min}$. The distribution data are collected with 100 crystals by using optical microscope. Single-crystal X-ray diffraction

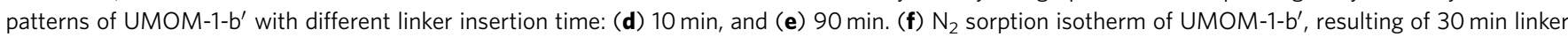
insertion reaction.

various MOMs, including solid, core-shell, double and triple matryoshka, and hollow single- and double-shell forms. Another marked difference between these and other hollow MOF crystals is single crystallinity. While other hollow MOFs often consist of aggregates of small crystals, the hollow MOFs presented here are the first case where single crystallinity is demonstrated by X-ray single-crystal diffraction. We expect that such examples of extreme self-assembly might open up new avenues to various forms of MOFs for synthesizing single crystalline multi-shelled hollow MOF with hierarchical porous materials with trimodal (for example, micro-/meso-/macro porous structures) pore system and highly advanced applications such as sequential drug delivery/release and biomimetic cascade catalysis. A good case for the latter can be found in nature, as exemplified by native pyruvate dehydrogenase (PDH2), a multifunctional catalytic machine with an icosahedral double-hollow structure. $\mathrm{PDH} 2$ is composed of three different enzymes that are distributed individually in two shells $\mathrm{s}^{50}$. This spatial separation is known to be crucial for the overall function of $\mathrm{PDH} 2$ and plays a key role in its catalytic behaviour. Mimicking such multi-component, macromolecular machines through surface modification or postsynthetic modification is a synthetic challenge for next-generation MOMs, and will further push the limit of self-assembly.

\section{Methods}

Materials. 5-hydroxy-1,3-benzenedicarboxylic acid (OH-mBDC; TCI), 1,4-diazabicyclo [2.2.2] octane (dabco; Sigma-Aldrich), 4,4'-bipyridyl (bpy; TCI) and $\mathrm{Cu}(\mathrm{OAc})_{2} \cdot \mathrm{H}_{2} \mathrm{O}$ (JUNSEI) were used without further purification. Methanol $(\mathrm{MeOH}), \mathrm{DMSO}$ and DMF were obtained from JUNSEI, and N,N'-dimethylacetamide was obtained from TCI.

Synthesis of UMOM-1. A MeOH $(4.0 \mathrm{ml})$ solution of OH-mBDC $(146.1 \mathrm{mg}, 0.802 \mathrm{mmol})$ was mixed with a $\mathrm{MeOH}(12.0 \mathrm{ml})$ solution of $\mathrm{Cu}(\mathrm{OAc})_{2} \cdot \mathrm{H}_{2} \mathrm{O}(160.0 \mathrm{mg}, 0.801 \mathrm{mmol})$ in a capped vial $(20 \mathrm{ml})$. After mixing,
$2.5 \mathrm{ml}$ of $\mathrm{N}, \mathrm{N}^{\prime}$-dimethylacetamide and $1.5 \mathrm{ml}$ of $\mathrm{MeOH}$ was added to this solution and then allowed the vial stand at room temperature. After 5 days, synthesized crystals were collected and dissolved in $20.0 \mathrm{ml}$ of $\mathrm{MeOH}$ (solution-A). In total, $3.0 \mathrm{ml}$ of solution-A was well mixed with $3.0 \mathrm{ml}$ of DMSO/DMF solution $(\mathrm{v} / \mathrm{v}=1: 1)$ and then allowed the vial stand at room temperature. After 1 day, blue crystals were recrystallized.

Synthesis of UMOM-2. The recrystallized UMOM-1 $(\sim 30.0 \mathrm{mg})$ was immersed in the $0.18 \mathrm{M}$ dabco solution with $10.0 \mathrm{ml}$ of DMSO/DMF mixture $(\mathrm{v} / \mathrm{v}=1: 1)$ and then left to react at room temperature. After 1 day, green crystals were obtained.

Synthesis of UMOM-1-a. The recrystallized UMOM-1 ( 30.0 mg) was immersed in $36.0 \mathrm{mM}$ dabco solution with $5.0 \mathrm{ml}$ of DMSO/DMF mixture $(\mathrm{v} / \mathrm{v}=1: 1)$ and then left to react at room temperature for time, $t(10 \mathrm{~min}<t<180 \mathrm{~min})$. The reacted crystals were rinsed with $5.0 \mathrm{ml}$ pure DMSO/DMF mixture $(\mathrm{v} / \mathrm{v}=1: 1)$ for three times.

Synthesis of UMOM-1-b'. UMOM-1-a was immersed in $5.0 \mathrm{ml}$ of $\mathrm{MeOH}$ at room temperature. After 1 day, blue hollow crystals were collected.

Synthesis of UMOM-1-b. In total, $2.0 \mathrm{ml}$ of solution-A and $2.0 \mathrm{ml}$ of DMSO/DMF solution $(\mathrm{v} / \mathrm{v}=1: 1)$ were carefully mixed and left to react at room temperature. After $4 \mathrm{~h}$, blue crystals were recrystallized. After decanting the solution, $5.0 \mathrm{ml}$ of $36.0 \mathrm{mM}$ dabco solution was added into the recrystallized crystals and then left to react at room temperature for $10 \mathrm{~min}$ and then rinsed the with $5.0 \mathrm{ml}$ pure DMSO/DMF mixture $(\mathrm{v} / \mathrm{v}=1: 1)$ for three times. After that, collected crystals were immersed in $3.0 \mathrm{ml}$ of DMSO/DMF mixture $(\mathrm{v} / \mathrm{v}=1: 1)$ and $3.0 \mathrm{ml}$ of solution- $\mathrm{A} / \mathrm{MeOH}$ mixture $(\mathrm{v} / \mathrm{v}=1: 2)$ and then left to react at room temperature for 1 day. After decanting the solution, $3.0 \mathrm{ml}$ of DMSO/DMF mixture $(\mathrm{v} / \mathrm{v}=1: 1)$ and $3.0 \mathrm{ml}$ of solution- $\mathrm{A} / \mathrm{MeOH}$ mixture $(\mathrm{v} / \mathrm{v}=1: 2)$ were added and well mixed with crystals then left to react at room temperature for 1 day.

Synthesis of UMOM-1-c. UMOM-1-b was immersed in $36.0 \mathrm{mM}$ dabco solution with $5.0 \mathrm{ml}$ of DMSO/DMF mixture $(\mathrm{v} / \mathrm{v}=1: 1)$ and then left to react at room temperature for $10 \mathrm{~min}$. The reacted crystals were rinsed with $5.0 \mathrm{ml}$ pure $\mathrm{DMSO} / \mathrm{DMF}$ mixture $(\mathrm{v} / \mathrm{v}=1: 1)$ for three times. 
a

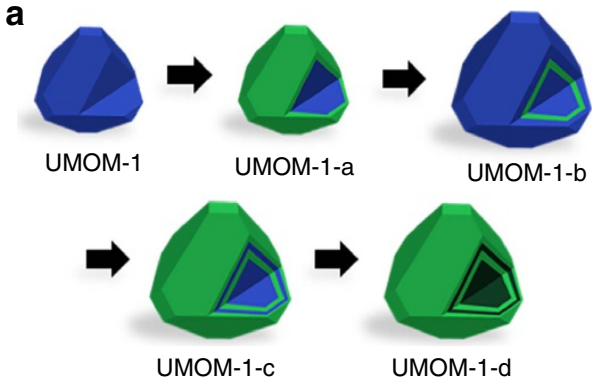

C

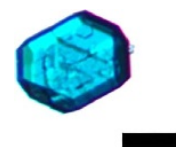

d

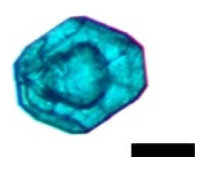

b

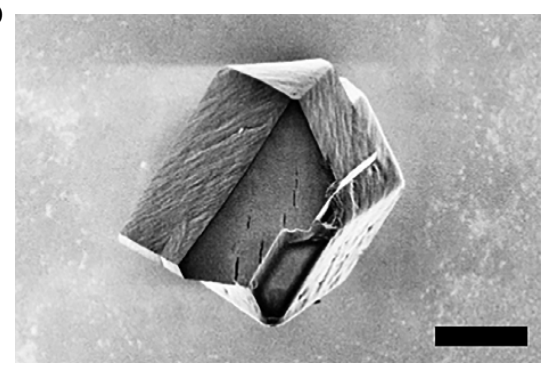

e

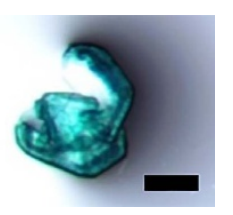

$\mathbf{f}$

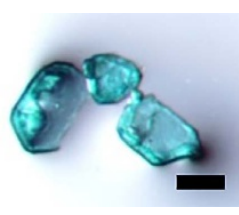

$\mathbf{h}$

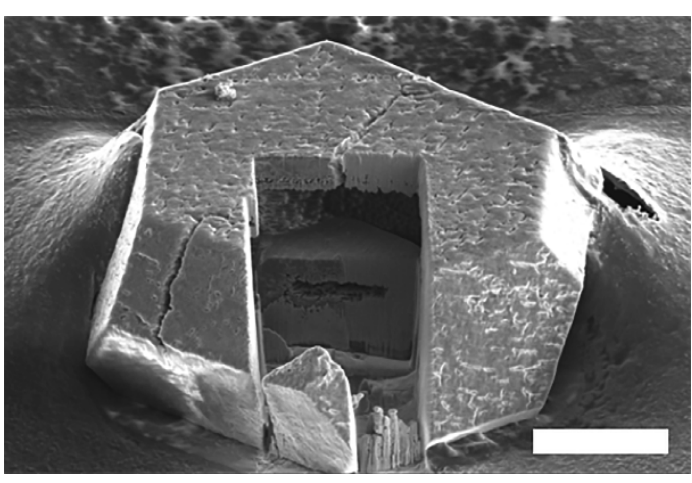

Figure 6 | Microscopic images of double-shell hollow UMOM-1-d. (a) The evolution of form in MOFs: from UMOM-1, UMOM-1-a, UMOM-1-b, UMOM-1-c and finally to UMOM-1-d. (b) SEM image of single crystal which is in epitaxial growth process of UMOM-1 on the crystal surface of UMOM-1-a. Scale bar, $50 \mu \mathrm{m}$. Optical microscopic images of (c) UMOM-1-b, (d) UMOM-1-d and (e,f) broken crystal of UMOM-1-d. Scale bar, 100 $\mu \mathrm{m}$ (c-f). FIB-SEM images of single crystal of UMOM-1-d: (g) before the milling and (h) after milling with $52^{\circ}$ rotation. Scale bar, $50 \mu \mathrm{m}(\mathbf{g}), 25 \mu \mathrm{m}(\mathbf{h})$.

Synthesis of UMOM-1-d. UMOM-1-c was immersed in $5.0 \mathrm{ml}$ of $\mathrm{MeOH}$ and then left to react at room temperature. After 1 day, blue double-shell hollow crystals were collected.

Characterization. X-ray synchrotron powder diffraction data were taken at the PAL, Republic of Korea. The well ground powder was packed into the $0.5 \mathrm{~mm}$ diameter of capillary (wall thickness: $0.01 \mathrm{~mm}$ ). Diffraction data were collected with ADSC Quantum-210 detector at two-dimensional (2D) supramolecular cyrstallography with a silicon (111) double-crystal monochromator. All data were collected at $298 \mathrm{~K}$ with a $150 \mathrm{~mm}$ of detector distance using synchrotron radiation $(\lambda=1.40009 \AA)$. The ADX programme $\mathrm{e}^{51}$ was used for data collection and the Fit2D programme (ESRF Internal Report, ESRF98HA01T, FIT2D V9.129 Reference Manual V3.1, 1998) was used for data convert a 2D diffraction image to a onedimensional diffraction pattern ${ }^{52}$. A single crystal of UMOM-1 coated with paratone-N oil and the diffraction data were collected at $298 \mathrm{~K}$ with ADSC Quantum-210 detector at 2D supramolecular cyrstallography with a silicon (111) double-crystal monochromator at the PAL, Republic of Korea. The ADSC Q210 ADX programme ${ }^{51}$ was used for data collection, and HKL3000 (ref. 53) was used for cell refinement, reduction and absorption correction. The single-crystal diffraction data of UMOM-2 and UMOM-1- $\mathrm{b}^{\prime}$ were collected at $296 \mathrm{~K}$ with $\mathrm{Mo} \mathrm{K} \alpha$ radiation using a Rigaku R-Axis Rapid II $(0.3 \mathrm{~mm}$ capillaries with wall thickness: $0.01 \mathrm{~mm}$ DMSO solvent). The Rapid Auto software (Rapid Auto software, R-Axis series, Cat. No. 9220B101, Rigaku Corporation) was used for data collection and processing. All crystal structures were solved by the direct method and were refined by full-matrix least-squares calculations using the SHELXL programme package ${ }^{54}$. NMR was performed on an Agilent FT-NMR spectrometer $(400 \mathrm{MHz})$. Ultraviolet-visible absorbance spectra were performed on Agilent Cary 5000. Thermogravimetric Analysis was performed on a TA instrument SDT Q600, heated from 25 to $600{ }^{\circ} \mathrm{C}$ under $\mathrm{N}_{2}$ atmosphere at a scan rate of $2{ }^{\circ} \mathrm{C} \mathrm{min}^{-1}$. Gas sorption isotherm was performed on Micromeritics ASAP 2020 instrument. Pore size distributions were obtained using oxide surface cylindrical model with a $\mathrm{N}_{2}$ isotherm. Field emission SEM images were obtained from Hitachi S- 4800 at an acceleration voltage of $5 \mathrm{kV}$. FIB-SEM images were obtained from FEI Helios $4850 \mathrm{HP}$ with current $80 \mathrm{pA}$ at $30 \mathrm{kV}$ and the FIB milling was performed with $\mathrm{Ga}$ ion beam current of $2.5-9.3 \mathrm{nA}$ at $30 \mathrm{kV}$.

Data availability. The X-ray crystallographic coordinates for structures reported in this Article have been deposited at the Cambridge Crystallographic Data Centre (CCDC), under deposition number CCDC 1500392 (UMOM-1), CCDC 1500390 (UMOM-2) and CCDC 1500391 (UMOM-1-b'). These data can be obtained free of charge from The Cambridge Crystallographic Data Centre via www.ccdc.cam.ac.uk/data_request/cif, all remaining data can be obtained from the corresponding authors on request.

\section{References}

1. Chaudhuri, R. G. \& Paria, S. Core/shell nanoparticles: classes, properties, synthesis mechanisms, characterization, and applications. Chem. Rev. 112, 2373-2433 (2012).

2. Shen, L. et al. Formation of nickel cobalt sulfide ball-in-ball hollow spheres with enhanced electrochemical pseudocapacitive properties. Nat. Commun. 6, 6694-6701 (2015).

3. Priebe, M. \& Fromm, K. M. Nanorattles or yolk-shell nanoparticles-what are they, how are they made, and what are they good for? Chem. Eur. J. 21, 3854-3874 (2015).

4. Lou, X. W., Archer, L. A. \& Yang, Z. Hollow micro-/nanostructures: synthesis and applications. Adv. Mater. 20, 3987-4019 (2008).

5. Yaghi, O. M. et al. Reticular synthesis and the design of new materials. Nature 423, 705-714 (2003).

6. Cook, T. R., Zheng, Y.-R. \& Stang, P. J. Metal-Organic frameworks and selfassembled supramolecular coordination complexes: comparing and contrasting the design, synthesis, and functionality of metal-organic materials. Chem. Rev. 113, 734-777 (2013).

7. Liang, H.-P. et al. Pt hollow nanospheres: facile synthesis and enhanced electrocatalysts. Angew. Chem. Int. Ed. 43, 1540-1543 (2004). 
8. Qi, J. et al. Multi-shelled $\mathrm{CeO}_{2}$ hollow microspheres as superior photocatalysts for water oxidation. Nanoscale 6, 4072-4077 (2014).

9. Wang, Y. et al. Designed functional systems from peapod-like Co@carbon to $\mathrm{Co}_{3} \mathrm{O}_{4} @$ carbon nanocomposites. ACS Nano 4, 4753-4761 (2010).

10. Lee, I., Joo, J. B., Yin, Y. \& Zaera, F. A yolk@shell nanoarchitecture for Au/ $/ \mathrm{TiO}_{2}$ catalysts. Angew. Chem. Int. Ed. 50, 10208-10211 (2011).

11. Martinez, C. J., Hockey, B., Montgomery, C. B. \& Semancik, S. Porous tin oxide nanostructured microspheres for sensor applications. Langmuir 21, 7937-7944 (2005).

12. Lai, X. et al. General synthesis and gas-sensing properties of multiple-shell metal oxide hollow microspheres. Angew. Chem. Int. Ed. 50, 2738-2741 (2011).

13. Bai, Z. et al. Fluorescent pH sensor based on $\mathrm{Ag@SiO} \mathrm{S}_{2}$ core-shell nanoparticle. ACS Appl. Mater. Interfaces 5, 5856-5860 (2013).

14. Lou, X. W., Wang, Y., Yuan, C., Lee, J. Y. \& Archer, L. A. Template-free synthesis of $\mathrm{SnO}_{2}$ hollow nanostructures with high lithium storage capacity. Adv. Mater. 18, 2325-2329 (2006).

15. Liu, N. et al. A yolk-shell design for stabilized and scalable Li-ion battery alloy anodes. Nano Lett. 12, 3315-3321 (2012).

16. Liu, J., Xia, H., Xue, D. \& Lu, L. Double-shelled nanocapsules of $\mathrm{V}_{2} \mathrm{O}_{5}$-based composites as high-performance anode and cathode materials for $\mathrm{Li}$ ion batteries. J. Am. Chem. Soc. 131, 12086-12087 (2009).

17. Ren, H. et al. Multishelled $\mathrm{TiO}_{2}$ hollow microspheres as anodes with superior reversible capacity for lithium ion batteries. Nano Lett. 14, 6679-6684 (2014).

18. Chen, J. et al. Gold nanocages: bioconjugation and their potential use as optical imaging contrast agents. Nano Lett. 5, 473-477 (2005).

19. Gao, J. et al. Multifunctional yolk - shell nanoparticles: a potential MRI contrast and anticancer agent. J. Am. Chem. Soc. 130, 11828-11833 (2008).

20. Ayala-Orozco, C. et al. Au nanomatryoshkas as efficient near-infrared photothermal transducers for cancer treatment: benchmarking against nanoshells. ACS Nano 8, 6372-6381 (2014).

21. Kim, T. et al. Mesoporous silica-coated hollow manganese oxide nanoparticles as positive $\mathrm{T}_{1}$ contrast agents for labeling and MRI tracking of adipose-derived mesenchymal stem cells. J. Am. Chem. Soc. 133, 2955-2961 (2011).

22. Furukawa, S., Reboul, J., Diring, S., Sumida, K. \& Kitagawa, S. Structuring of metal-organic frameworks at the mesoscopic/macroscopic scale. Chem. Soc. Rev. 43, 5700-5734 (2014).

23. Li, T., Sullivan, J. E. \& Rosi, N. L. Design and preparation of a core-shell metalorganic framework for selective $\mathrm{CO}_{2}$ capture. J. Am. Chem. Soc. 135, 9984-9987 (2013).

24. Koh, K., Wong-Foy, A. G. \& Matzger, A. J. MOF@MOF: microporous coreshell architectures. Chem. Commun. 2009, 6162-6164 (2009).

25. Lee, H. J., Cho, W. \& Oh, M. Advanced fabrication of metal-organic frameworks: template-directed formation of polystyrene@ZIF-8 core-shell and hollow ZIF-8 microspheres. Chem. Commun. 48, 221-223 (2012).

26. Li, A.-L. et al. Controllable synthesis of metal-organic framework hollow nanospheres by a versatile step-by-step assembly strategy. CrystEngComm. 15, 3554-3559 (2013)

27. Pang, M. et al. Synthesis and integration of Fe-soc-MOF cubes into colloidosomes via a single-step emulsion-based approach. J. Am. Chem. Soc. 135, 10234-10237 (2013).

28. Peng, L. et al. Hollow metal-organic framework polyhedra synthesized by a $\mathrm{CO}_{2}$-ionic liquid interfacial templating route. J. Colloid Interface Sci. 416, 198-204 (2014)

29. Hirai, K. et al. Diffusion-coupled molecular assembly: structuring of coordination polymers across multiple length scales. J. Am. Chem. Soc. 136, 14966-14973 (2014)

30. Kim, H. et al. Single crystalline hollow metal-organic frameworks: a metalorganic polyhedron single crystal as a sacrificial template. Chem. Commun. 51, 3678-3681 (2015).

31. Ameloot, R. et al. Interfacial synthesis of hollow metal-organic framework capsules demonstrating selective permeability. Nat. Chem. 3, 382-387 (2011).

32. Carné-Sánchez, A., Imaz, I., Cano-Sarabia, M. \& Maspoch, D. A spray-drying strategy for synthesis of nanoscale metal-organic frameworks and their assembly into hollow superstructures. Nat. Chem. 5, 203-211 (2013).

33. Zhang, Z. et al. Well-defined metal-organic framework hollow nanocages. Angew. Chem. Int. Ed. 53, 429-433 (2014).

34. Hu, P., Morabito, J. V. \& Tsung, C.-K. Core-shell catalysts of metal nanoparticle core and metal-organic framework shell. ACS Catal. 4, 4409-4419 (2014).

35. Lu, G. et al. Imparting functionality to a metal-organic framework material by controlled nanoparticle encapsulation. Nat. Chem. 4, 310-316 (2012).

36. Na, K., Choi, K. M., Yaghi, O. M. \& Somorjai, G. A. Metal nanocrystals embedded in single nanocrystals of MOFs give unusual selectivity as heterogeneous catalysts. Nano Lett. 14, 5979-5983 (2014).

37. Qi, J. et al. Multi-shelled hollow micro-/nanostructures. Chem. Soc. Rev. 44, 6749-6773 (2015)

38. Perry, IV J. J., Perman, J. A. \& Zaworotko, M. J. Design and synthesis of metalorganic frameworks using metal-organic polyhedra as supermolecular building blocks. Chem. Soc. Rev. 38, 1400-1417 (2009).
39. Li, J.-R. \& Zhou, H.-C. Bridging-ligand-substitution strategy for the preparation of metal-organic polyhedra. Nat. Chem. 2, 893-898 (2010).

40. Guillerm, V. et al. A supermolecular building approach for the design and construction of metal-organic frameworks. Chem. Soc. Rev. 43, 6141-6172 (2014)

41. Kim, D., Liu, X. \& Lah, M. S. Topology analysis of metal-organic frameworks based on metal-organic polyhedra as secondary or tertiary building units. Inorg. Chem. Front 2, 336-360 (2015).

42. Chun, H., Jung, H. \& Seo, J. Isoreticular metal-organic polyhedral networks based on 5-connecting paddlewheel motifs. Inorg. Chem. 48, 2043-2047 (2009)

43. Wang, H.-N. et al. Stepwise assembly of metal-organic framework based on a metal-organic polyhedron precursor for drug delivery. Chem. Commun. 47, 7128-7130 (2011).

44. Burnett, B. J., Barron, P. M., Hu, C. \& Choe, W. Stepwise synthesis of metalorganic frameworks: replacement of structural organic linkers. J. Am. Chem. Soc. 133, 9984-9987 (2011).

45. Burnett, B. J. \& Choe, W. Sequential self-assembly in metal-organic frameworks. Dalton Trans. 41, 3889-3894 (2012)

46. Deria, P. et al. Beyond post-synthesis modification: evolution of metal-organic frameworks via building block replacement. Chem. Soc. Rev. 43, 5896-5912 (2014).

47. Luebke, R. et al. The unique rht-MOF platform, ideal for pinpointing the functionalization and $\mathrm{CO}_{2}$ adsorption relationship. Chem. Commun. 48, 1455-1457 (2012).

48. Li, B. et al. Enhanced binding affinity, remarkable selectivity, and high capacity of $\mathrm{CO}_{2}$ by dual functionalization of a rht-type metal-organic framework. Angew. Chem. Int. Ed. 51, 1412-1415 (2012).

49. Schoedel, A., Ji, Z. \& Yaghi, O. M. The role of metal-organic frameworks in a carbon-neutral energy cycle. Nat. Energy 1, 16034 (2016).

50. Milne, J. L. S. et al. Molecular structure of a 9-MDa icosahedral pyruvate dehydrogenase subcomplex containing the E2 and E3 enzymes using cryoelectron microscopy. J. Biol. Chem. 281, 4364-4370 (2006).

51. Arvai, A. J. \& Nielsen, C. ADSC Quantum-210 ADX Program (Area Detector System Corporation, Poway, CA, USA, 1983).

52. Hammersley, A. P., Svensson, S. O., Hanfland, M., Fitch, A. N. \& Häusermann, D. Two-dimensional detector software: from real detector to idealised image or two-theta scan. High Press. Res. 14, 235-248 (1996).

53. Otwinowski, Z. \& Minor, W. Processing of X-ray diffraction data collected in Oscillation mode. Methods Enzymol. 276, 307-326 (1997).

54. Sheldrick, G. M. A short history of SHELX. Acta Cryst. Sect. A 64, 112-122 (2008).

\section{Acknowledgements}

The authors acknowledge PAL for 2D beamline use (2014-3rd-2D-024). This work was supported by National Research Foundation (NRF) of Korea (NRF-2014K1A3A1A09063072) and of Ulsan National Institute of Science and Technology. J.H.K. acknowledges the support from NRF of Korea (NRF-2013R1A1A2009307).

\section{Author contributions}

W.C. conceived and designed the project. J.H.K. provided the experimental advice and analysed the data. J.L. performed the experiments and analysed the data. All the authors contributed to the writing of the paper.

\section{Additional information}

Supplementary Information accompanies this paper at http://www.nature.com/ naturecommunications

Competing financial interests: The authors declare no competing financial interests

Reprints and permission information is available online at http://npg.nature.com/ reprintsandpermissions/

How to cite this article: Lee, J. et al. Evolution of form in metal-organic frameworks. Nat. Commun. 8, 14070 doi: 10.1038/ncomms14070 (2017).

Publisher's note: Springer Nature remains neutral with regard to jurisdictional claims in published maps and institutional affiliations.

This work is licensed under a Creative Commons Attribution 4.0 International License. The images or other third party material in this article are included in the article's Creative Commons license, unless indicated otherwise in the credit line; if the material is not included under the Creative Commons license, users will need to obtain permission from the license holder to reproduce the material. To view a copy of this license, visit http://creativecommons.org/licenses/by/4.0/ 